\title{
Fuzzy Controller for Simplified Model of Mobile Vehicle Steering Booster
}

doi:10.2478/mape-2021-0004

Date of submission to the Editor: 04/2021

Date of acceptance by the Editor: 05/2021

MAPE 2021, volume 4, issue 1, pp. 40-50

\section{Peter Koleda}

ORCID ID: 0000-0003-3996-2621

Technical University in Zvolen, Slovak Republic

L’ubomír Naščák

Technical University in Zvolen, Slovak Republic

Mária Hrčková

Technical University in Zvolen, Slovak Republic

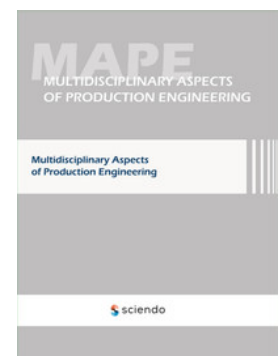

Róbert Magdolinič

Slovak Republic

\section{INTRODUCTION}

The diversity of mechatronic systems not always allows application of control systems based on classic concepts and simple controllers. Modern systems use elements of artificial intelligence, genetic algorithms and neural networks that can learn and apply the changed operating conditions. The value of control action is often complicated to compute, mainly if this value depends on various input conditions. Furthermore, such computing step is time-consuming and difficult for processor processing. Controllers based on fuzzy logic have led to more effective techniques since their introduction, whereby those controllers specify output manipulating variable on the base of rule set and membership function of input variables into fuzzy sets. Fuzzy systems combine human knowledge and computer information; they are universal function approximators (Yager and Filey, 1994), although, there must exist a system that approximates it to any degree of accuracy (Kasabov, 1998).

Fuzzy sets can be considered as a means, which allows mathematical describing of casual terms and working with them. It is a mathematical write of inaccuracy as a side-effect of complicated systems, that cannot be exactly determined, or the mathematical describing is too difficult. Because of this, fuzzy logic is often used in systems where the human factor has its importance (Holiš, 2001). Finding of optimal parameters of fuzzy controller in automation by the inference mechanism resulted in using Mamdami inference mechanism (MinMax method) and Larsen inference (Prod-Max method) (Modrlák, 2004; Pivonka, 2003). However, there are various classes of fuzzy systems models, whereby their basic division can be into Linguistic Models (Mamdami type) and the Takagi-Sugeno-Kang fuzzy models (Yager and Filey, 2003). In the work by Kasabov (1998) there is noted that fuzzy control systems were used as one of the first industrial fuzzy systems and were implemented for cement kiln control 
in the United Kingdom in the early 1970s. Currently fuzzy control systems are developed for many not only industrial applications.

Automatic control of vehicle was the subject of intense research last years ago (Cordón et al., 2004). The most common vehicle navigation principles are based on the principle of inductive navigation, which uses a network of buried cables or optical navigation principles. In both cases, the trajectory tracking device provides sufficient information for the controller. The most commonly used controller was classic PID controller. Recently, image processing techniques are largely developed, which in this area, using optoelectronic sensors, provide additional benefits such as obtaining information about the surroundings autonomously navigated vehicle. That allows creating automated systems to avoid collisions. In the work by Cardoso et al. (1994) the authors used a fuzzy controller for controlling the vehicle speed and steering angle calculation. In automatic vehicle guidance system was used the CCD camera as a sensor.

Modern electronic control of automatic transmission calculates the shift characteristics from a large amount of information that continuously describe the current operating status and driving situation. Control program, which works with regards to the driving resistance, recognizes driving resistances such as driving uphill, drive with a trailer or driving against the wind. According to the speed, throttle valve position, engine speed, and acceleration of the vehicle, control unit of automatic transmission calculates the driving resistance and, on the base of the result, determines the shift point (Jan and Ždánský, 2001). Fuzzy control systems have been potential candidates for the control of non-linear, timeunstable and complex systems. Anti-lock brake system (ABS), which is a nonlinear system, is difficult for controlling by the conventional methods. To improve the control, if the braking force is applied to the wheel system, longitudinal relationship of friction between the vehicle and the control are increasing rapidly. Brakes work because friction reactions actuate against the traction wheels. Larger slip is created by a lack of friction, greater braking force is important for constant momentum of the vehicle. Intelligent control method based on fuzzy logic is very useful for this type of nonlinear system. A typical ABS system releases the brakes for a short time and then re-activates them when the wheel starts to accelerate, according to the size of the grip of the wheel. In the literature (Wang et al., 2013), intelligent fuzzy ABS control system was proposed to adjust the slip effect for different types of road surfaces. One fuzzy control system was used for calculating the optimal braking torque equally to front and rear wheels and another fuzzy control system was used for calculating the size of slip and torque for different road types. Problem of finding the optimal parameters of defuzzifiers can solve a learning algorithm (Esogbue and Song, 2003). 


\section{EXPERIMENTAL MODEL}

The experimental model represents simplified model of automobile steering booster (Fig. 1, Fig. 2). It is composed of power supply unit, electromechanical system with stepper motors from a graphical unit, power driver, and processor controlling system. For control of mechatronic experimental model, the fuzzy logic is used.
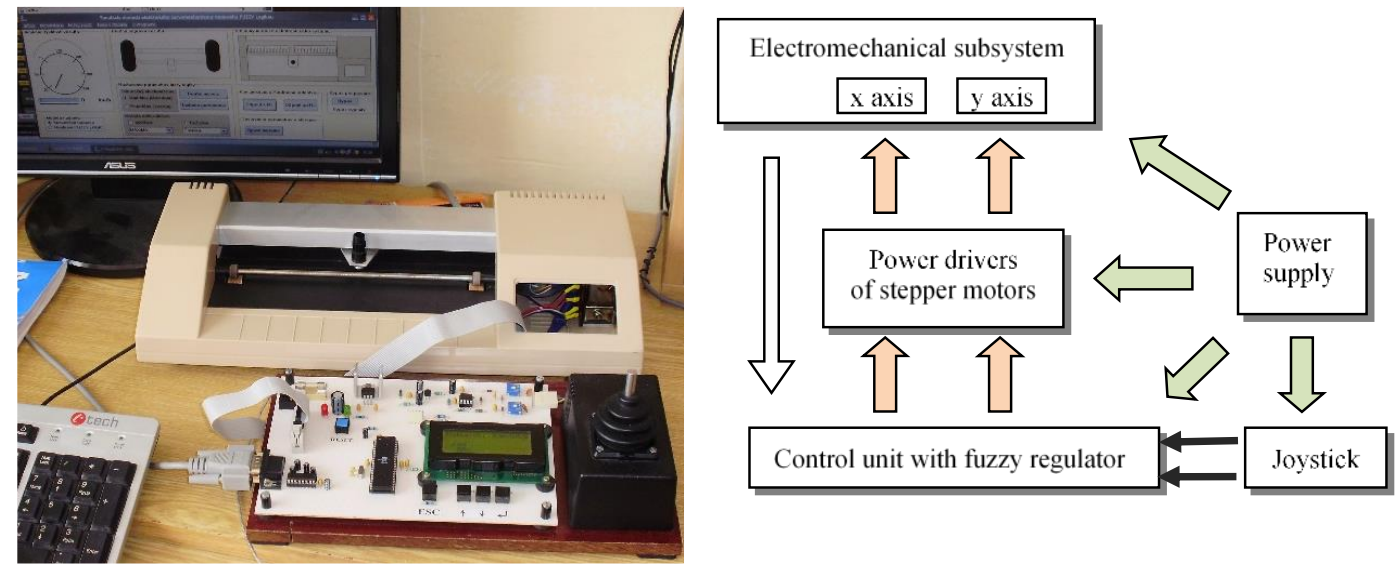

Fig. 1 Experimental model and its structure

The coordinate drawing system is useable as a system representing the function of electromechanical steering booster in a car. The part of model with slider represents the steering rack of the front axle of the car and driving rod represents the car speed.

In the tasks of controlling microcomputer, monolithic microcomputer ATMEGA32L by Atmel has been selected. The core of the system is complemented by an external reset circuit. For the creation of control software, we preferred the programming language $\mathrm{C}$.

The joystick JC 200 with inductive built-in circuit substituted the function of steering wheel. It generates analogue signals according to the angle deviation of joystick arm. This system allows independent controlling of two actuators in two axes without feedback. Actuators are controlled only regarding information from joystick that gives desired values. Therefore, it is an open numerical system with controlled speed in the first axis and with controlled position in the second axis.

The control of the first stepper motor, which represents the speed of the vehicle, by joystick, lies in sensing of the joystick position value and formatting of this value into speed. More specifically, joystick controls the acceleration engine. This means that the more the joystick amplitude accrues, the more the delay time of each control winding switching decreases. Indeed, the delay time is limited with the cut-off frequency of stepper motor Problems arose with the establishment of a transfer value of the joystick into the engine speed. This function visually appeared as a quadratic dependence and therefore it was necessary to create the inverse function to the motor had constant acceleration at each deviation. The task was slightly simplified and inverse function was 
constructed as a linear fractional function, where, compared to inverse quadratic function, the implementation of the system has apparently unrecognizable differences, what saved the computation time.

The control of the second stepper motor, which represents the location of rotation axle of mobile machine by joystick is designed by other means. At the joystick deviation the motor positions the slider into corresponding position and at its releasing the motor moves the slider back to the starting position. Therefore, the control program is designed so that the difference of deviations values is converted to the number of motor steps. One deviation is sensed before the positioning and the other after adjusting positions. In addition, to improving the efficiency, the motor speed is increased gradually, not constant at the slider positioning. At the programming of the acceleration of both motors, their acceleration rate had to be considered.

General control principle, as is described in the previous paragraphs, was limited to sensing the joystick deviation angle, sensing the moment value was not included to the control algorithm. In the Figure 2 the structure of fuzzy regulator is displayed. Joystick deviation and vehicle speed represent the inputs variables. Control system replaces data fields so that the supporting moment value for servomotor of the electromechanical steering booster is calculated on the basis of the joystick deviation and vehicle speed values.

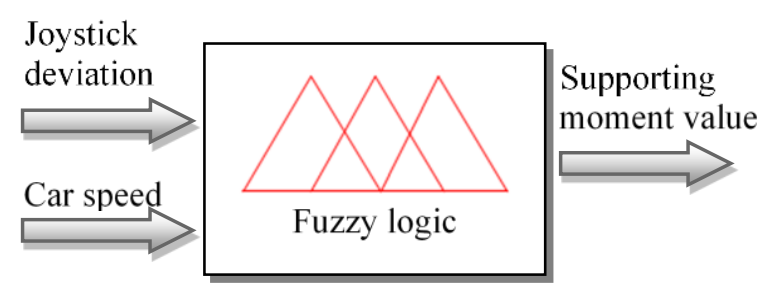

Fig. 2 Structure of fuzzy controller

The structure of fuzzy logic block is shown in the Fig. 3.

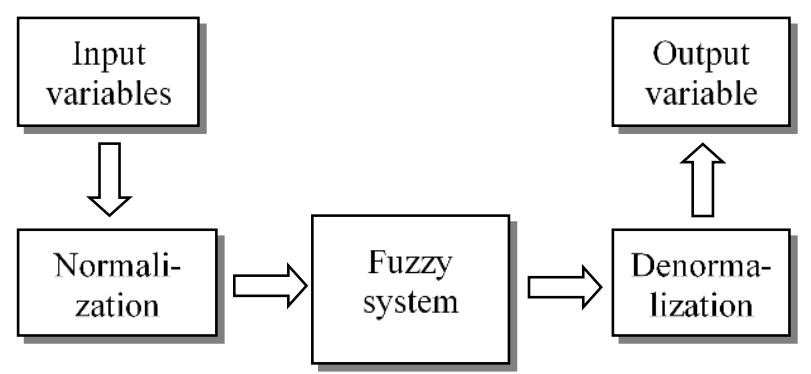

Fig. 3 Structure of fuzzy logic

In the normalization block the input variables were converted into normalized universes. Conversion was programmed as a linear functional dependence, where the values of the input variables were mapped into the range $<0,100>$. This block is exactly the opposite of the denormalization operation. Fuzzy 
system block represents a set of all control algorithms and its structure is shown in the Fig. 4.

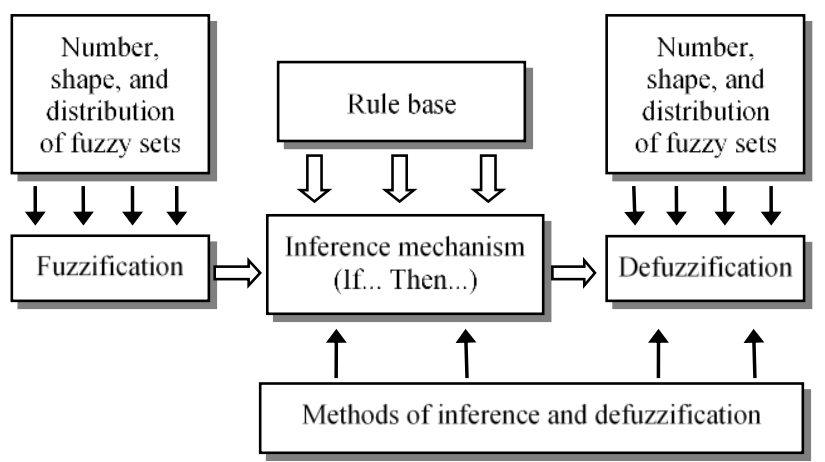

Fig. 4 Structure of fuzzy system

We implemented triangular and rectangular shapes of fuzzy sets. Explicitly realized selection of all relevant parameters (shape and number of fuzzy sets and their distribution) enters into fuzzification block, where the appropriate input values are fuzzificated.

\section{RESULTS}

The goal of experiment is to optimize the parameters of fuzzy regulator for computing the supporting torque value of electromechanical steering booster in the car or mobile machine. Fuzzy logic contains a lot of variables, whose combinations have to be correctly interpreted and evaluated. The application was designed in order to select only relevant parameters useable for optimization. Various shapes and number of fuzzy sets and their distribution on particular universes were simulated.

The problem may be to evaluate the interpretability of fuzzy modeling. There are several taxonomies based on an assessment of complexity and semantics. By their overlapping, the taxonomy can be divided into four groups (Gacto et al. 2011):

- complexity at the rule base level (number of rules, conditions),

- complexity at the fuzzy partition level (number of functions, features),

- semantics at the rule base level (consistency of rules, rule structure),

- semantics at the fuzzy partition level (completeness, normalization, distinguishability).

\section{Experiment 1}

Both programmed methods of inference mechanism, the Min-Max method and the Prod-Max method, had almost the same results. At defuzzification, the center methods appeared as better. Although, the methods of maximum are not complicated for computing, they are not enough suitable for designed application. Using these methods, function characteristics were changed only in 
some discrete points (Fig. 5) what would have a fatal effect on car steering system.

The centre methods had more favourable effect. The method of surface centre accounted smoother non-fractional functions in some regions. Marginal regions of universe are the disability of this method. They cannot be reached if the enhanced defuzzification method of surface centre is not used.

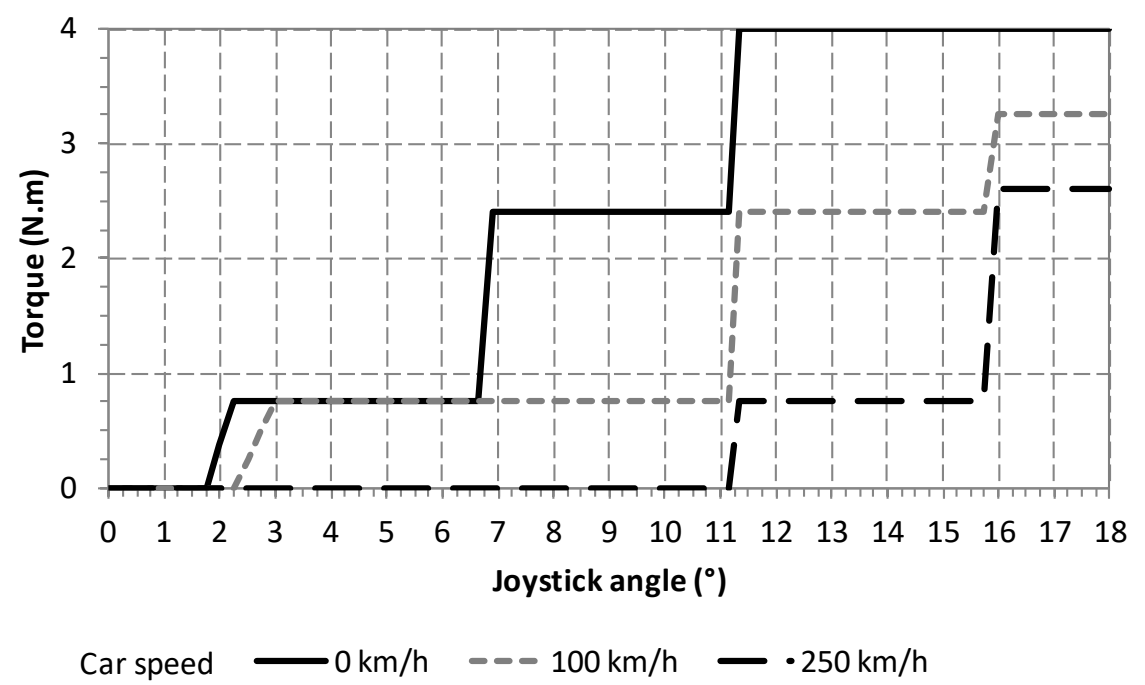

Fig. 5 Dependence of torque on joystick angle using methods of maximum

This method computes the courses of universe out of the universe range and consequently computes the centre of enhanced universe. Furthermore, this method is difficult for computing performance, what limited its using in proposed application. The most decisive results gave the maximum centre method.

By experimenting with four fuzzy sets on input universes (Fig. 6) and with five sets on output universes (Fig. 7 and 8), we found acceptable solution.

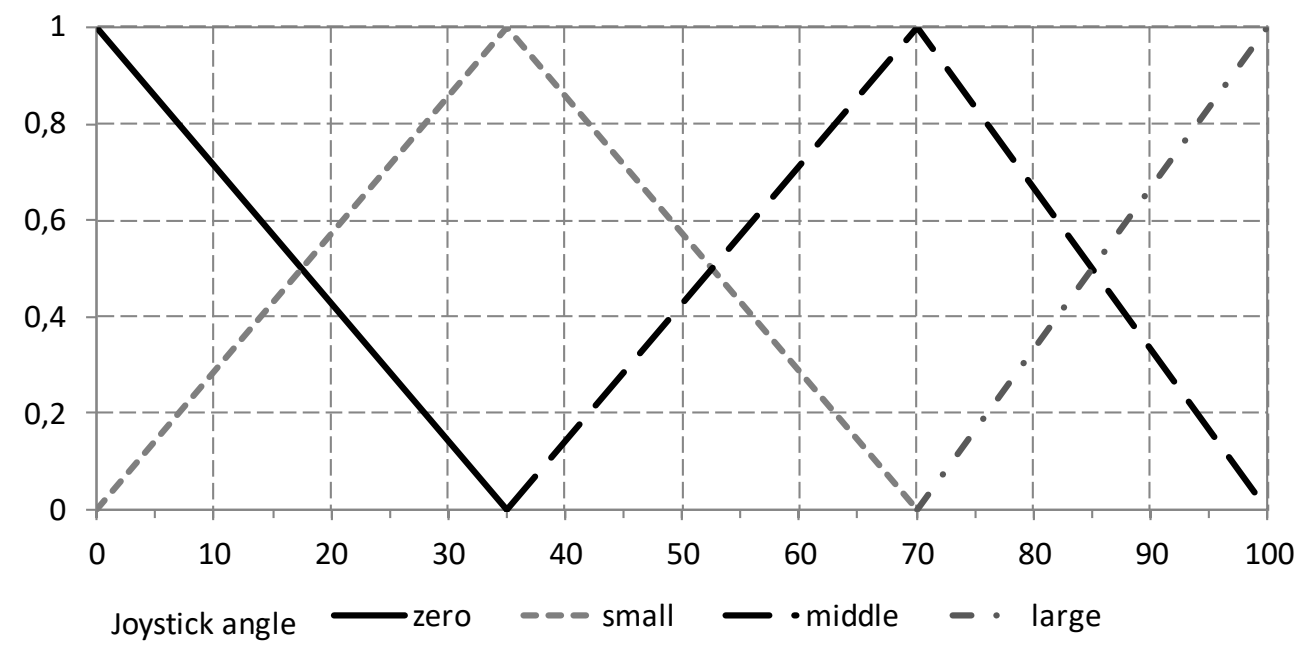

Fig. 6 Fuzzy sets for the joystick angle deviation 


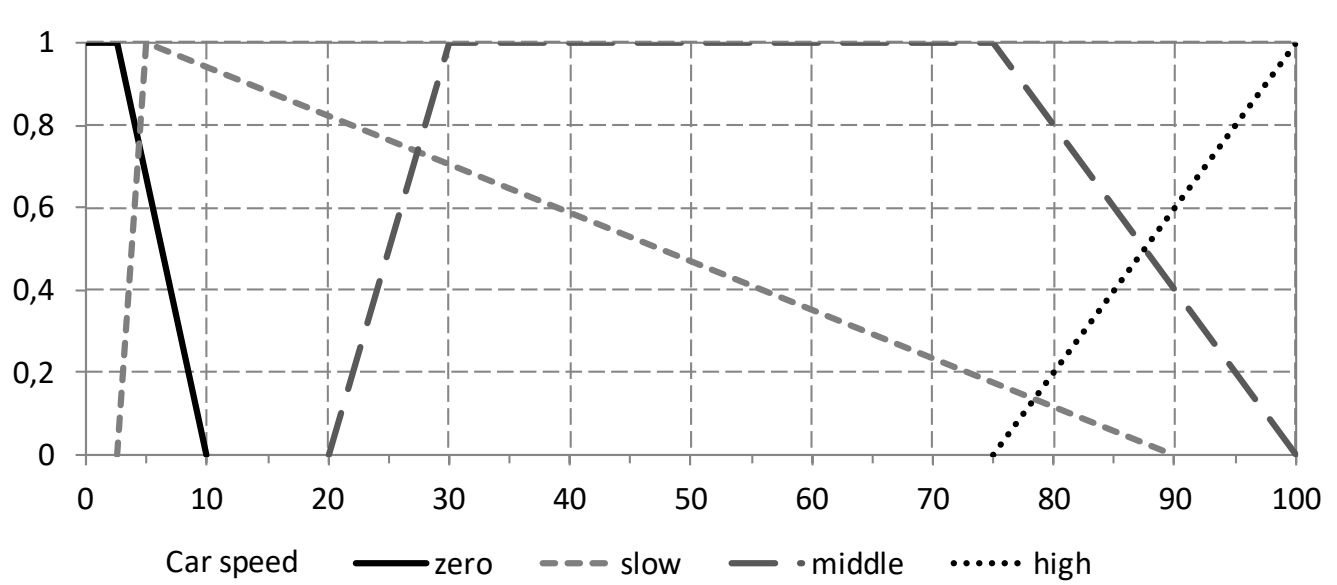

Fig. 7 Fuzzy sets for the speed of car

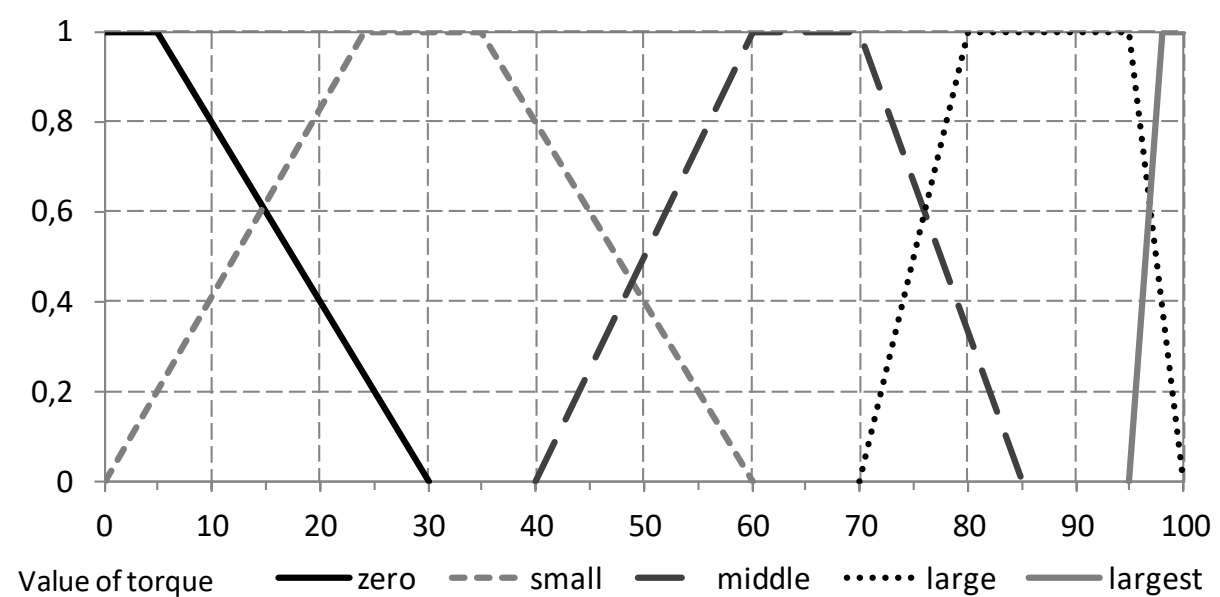

Fig. 8 Fuzzy sets for supporting torque

It appeared that the influence of fuzzy sets distribution on output universes was stronger at generation of resultant functions. The distribution of fuzzy sets on input universes had influence on tuning of functions in specified area. Inscribed facts are strong dependent on concrete situation and it is hard to generalize them for using in fuzzy system design. The rule set (Table 1) had the most important influence on output function courses, whereby there is defined the whole control strategy.

Described shapes and distributions of fuzzy sets on universes with the rule set gave the function of supporting torque dependent on the angle deviation of joystick at variable values of car speed, what is shown in the Fig. 9.

Table 1 Rule set for Experiment 1

\begin{tabular}{|c|c|c|c|c|c|}
\hline \multicolumn{2}{|c|}{} & \multicolumn{4}{|c|}{ Joystick angle } \\
\cline { 3 - 6 } & zero & small & middle & large \\
\hline \multirow{3}{*}{$\circlearrowleft$} & zero & zero & small & largest & largest \\
\cline { 2 - 6 } & slow & zero & small & middle & largest \\
\cline { 2 - 6 } & middle & zero & zero & small & large \\
\cline { 2 - 6 } & high & zero & zero & small & middle \\
\hline
\end{tabular}




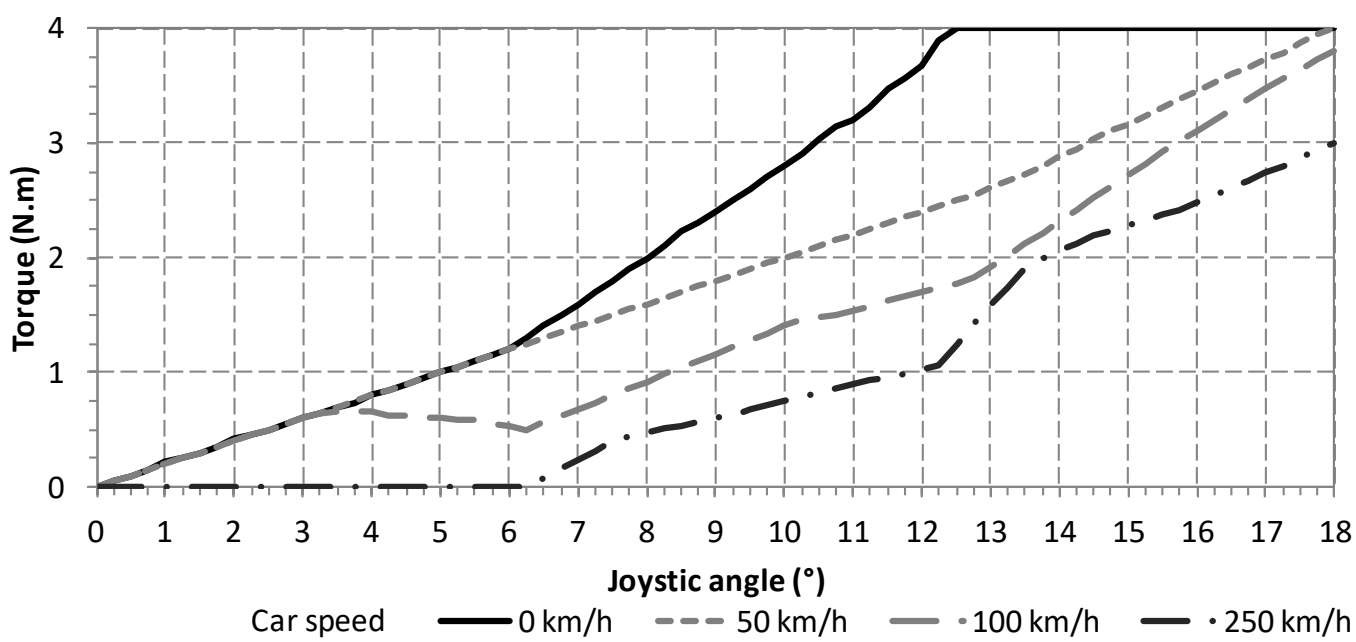

Fig. 9 Dependence of torque on joystick angle

\section{Experiment 2}

This experiment was performed with five terms on input universes (Fig. 10 and 11) and with six terms on output universes (Fig. 12). In this case, distribution of fuzzy sets on the second input and output universe was uniform with fuzzy sets in the shape of triangle.

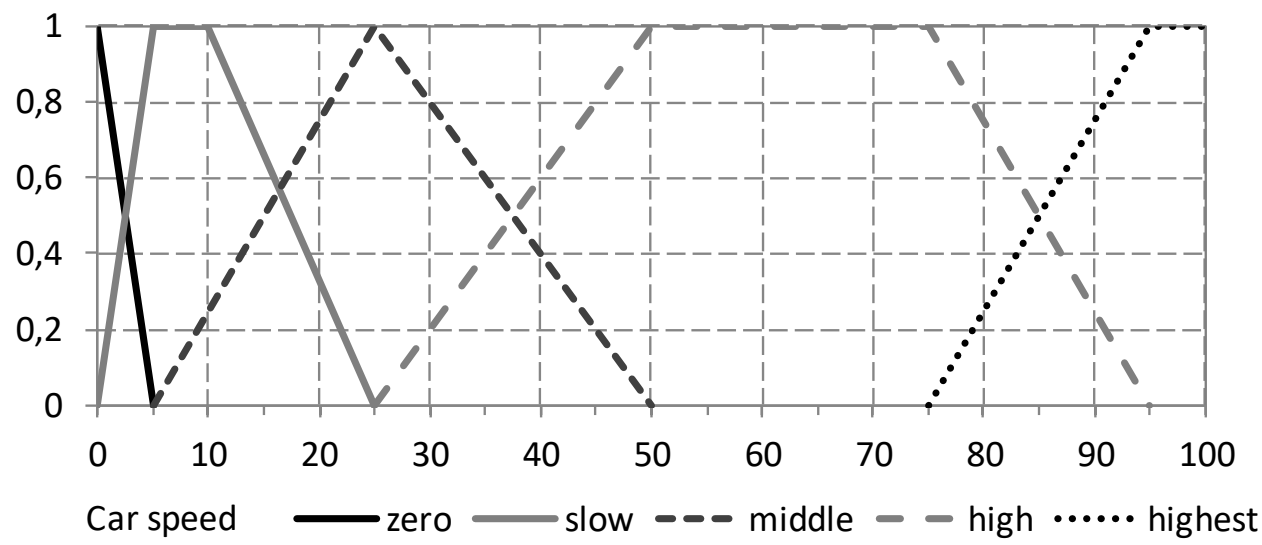

Fig. 10 Five fuzzy sets for speed of car

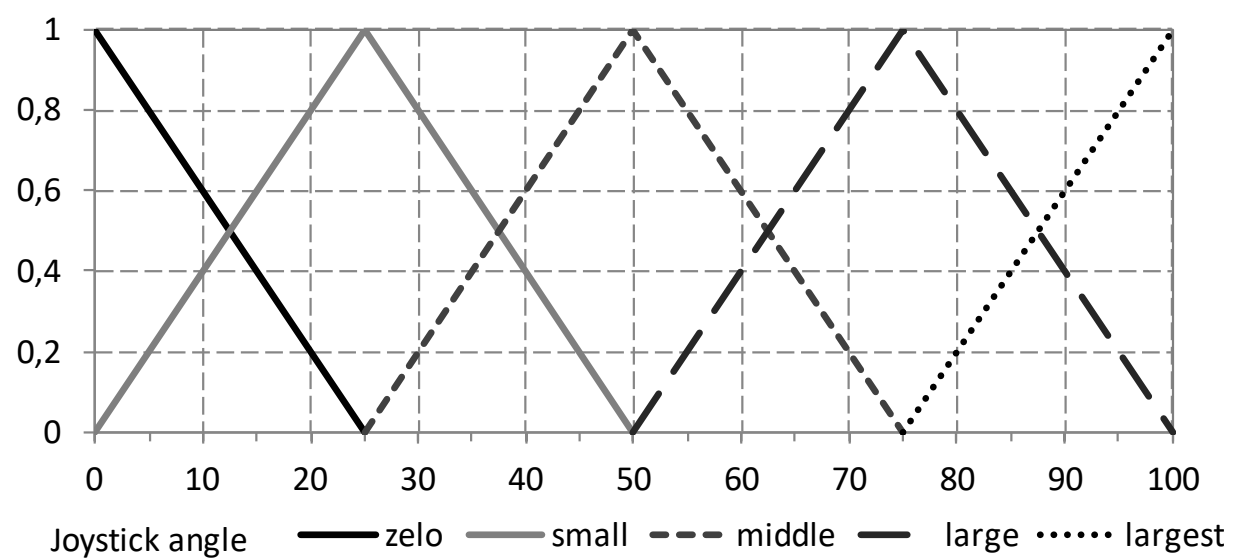

Fig. 11 Five fuzzy sets for joystick angle 


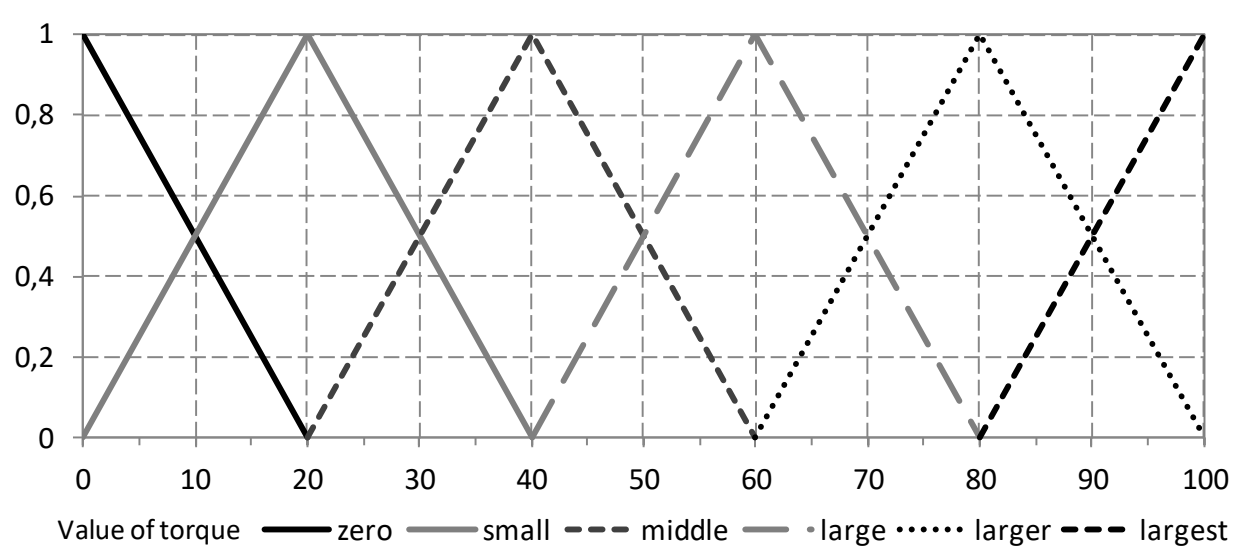

Fig. 12 Six fuzzy sets for supporting torque

Bigger count of input terms caused changes in rule set (Table 2) and allowed bigger variability of control strategy.

Table 2 Rule set for Experiment 2

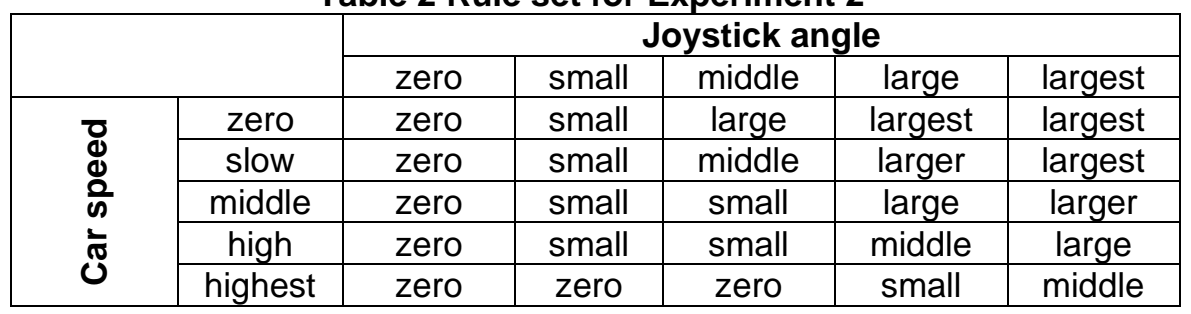

Enhancement of count of terms on input and output universes gave more satisfactory results. The function of supporting torque dependent on joystick angle deviation for variable car speeds is shown in the Fig. 13.

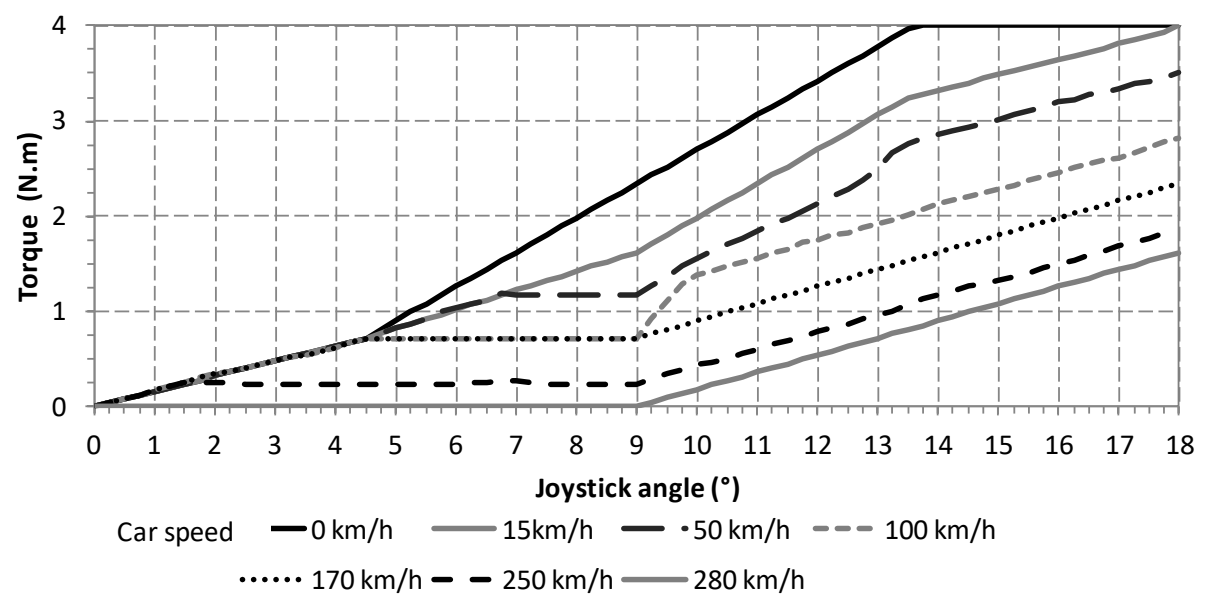

Fig. 13 Dependence of torque on joystick angle

\section{CONCLUSION}

Parameters of fuzzy logic used for achievement of described results are not optimal for all systems and they should be tuned on a real device. Moreover, there are problems with correct harmonization and adjusting of all variable parameters. The methods of soft programming, using neural network or evolution strategy for computation of optimal parameters, can solve this problem 
(Kecman 2001; Pradhan et al. 2006). At fuzzy system design and fuzzy sets defining, a lot of authors propose to use genetic algorithms that are able to adapt and find optimal solution for fuzzy sets distribution. There are several approaches that tune fuzzy sets and rule base using genetic algorithms. The most prominent types of genetic fuzzy systems are genetic fuzzy rule-based systems (Cordón 2004). Beside this, other communication protocol for superior control system of experimental model could bring better results (Koleda and Naščák 2009).

Experimental system had only two inputs: the car speed and angle deviation of joysticks. By addition of new input universe, representing the load of car or the load of front axle, described system could be more universal and used in cars of different types and with variable load.

Inscribed courses of functions are consistent with courses of functions in data arrays used in SKODA cars. Great advantage of fuzzy logic in that system is the variability of curves determining the value of supporting torque. These curves are continually changed according to the speed of car and they are not given for some discrete intervals only. In this article, fuzzy logic is used for approximation of non-linear functions. The non-linear system of data arrays, absence of a simple mathematical model, and suitable linguistic describing of system based on driver logic and intuition designate the using of fuzzy logic for controlling of examined mechatronic system.

\section{ACKNOWLEDGEMENTS}

The paper was written within the KEGA 006STU-4/2021 Project: "Progressive form of interdisciplinary education and supporting the subject-specific study development at universities“.

\section{REFERENCES}

Cardoso, F., et al. (1994). Fuzzy logic steering controller for a guided vehicle. Proceeding of the 7th Mediterranean Electrotechnical Conference, (Lisboa, Portugal), 711-714.

Cordón, O., Gomide, F., Herrera, F., Hoffmann, F., Magdalena, L. (2004). Ten years of genetic fuzy systems: current framework and new trends. Fuzzy sets and systems, 141, 5-31.

Esogbue, A. O., Song. Q. (2003). On Optimal Defuzzification and Learning Algorithms: Theory and Applications. Fuzzy Optimization and Decision Making, 2(4), 283296.

Gacto, M. J., Alcalá, R., Herrera, F. (2011). Interpretability of linguistic fuzzy rule-bases systems: An overview of interpretability measures. Information Sciences, 181. 4340-4360.

Holiš, M. (2001). Using of Neural-Fuzzy Systems for Control of Continual Processes in Indeterminacy Conditions. Thesis, Slovak Technical University.

Jan, Z., Ždánský B. (2001). Automobiles 2: Gears. Brno, Czech Republic: Avid.

Kasabov, N. K. (1998). Foundations of Neural Networks, Fuzzy Systems and Knowledge Engineering. London, England: MIT Press.

Kecman, V. (2001). Learning and soft computing: support vector machines, neural networks and fuzzy logic models. London, England: MIT Press. 
Koleda, P., Naščák, L'. (2009). Development and using of communication by means of Ethernet. In Perspective technology and materials for technical applications (pp. 116-121). Trnava, Inweld consulting.

Modrlák, O. (2004). Fuzzy Control and Regulation. Liberec, Czech Republic: TU Liberec.

Pivonka, P. (2003). Higher Forms of Control. Brno, Czech Republic: VUT.

Pradhan, S. K., Parhi, D. R., Panda, A. K. (2006). Neuro-fuzzy technique for navigation of multiple mobile robots. Fuzzy Optimization and Decision Making, 5(3), 255-288

Wang, D., Zeng, X., Keane, J. (2013). A simplified structure evolving method for Mamdami fuzzy system identification and its application to thigh-dimensional problems. Information Sciences, 220, 110-123.

Yager, R. R., Filev, D. P. (1994). Essentials of Fuzzy Modeling and Control. England: John Wiley \& Sons.

\begin{abstract}
The article is aimed on the possibility of fuzzy control of an experimental device that represents the simplified model of automobile steering booster. The main part of model consists of a graphical unit illustrating the control of speed and supporting torque. Based on the structure of fuzzy controller, various counts and configurations of fuzzy sets were defined, whereby the relevant results were achieved by their application on controlled system.
\end{abstract}

Keywords: Automobile Booster, Fuzzy Sets, Fuzzy Controller, Speed Control 